\title{
Regression - yet another clustering method
}

\author{
Piotr Gawrysiak, Henryk Rybinski, Michał Okoniewski \\ \{gawrysia, hrb, okoniews\} @ii.pw.edu.pl \\ Institute of Computer Science, Warsaw University of Technology \\ ul. Nowowiejska 15/19, 00-665 Warsaw, Poland
}

\begin{abstract}
The paper contains description of a new clustering methodology that partitions data set into clusters, such that regression indetermination coefficient for data from each cluster is minimized. A clustering algorithm that realizes this methodology with genetic programming approaches, as well as, some experimental results are presented. The application of the algorithm for planning cellular telephone networks is discussed.
\end{abstract}

Keywords: clustering, regression, data mining, cellular telephone networks

\section{Introduction}

Regression is a very robust and widely used method for data analysis. Its applications range from assessing experimental data, through mathematical and econometric model building to advanced data mining, mainly as one of the methods of estimating a relationship between some attributes. While being effective and relatively simple method, regression can be applied only for data that are internally consistent. This means that only one function should exists that links attributes, or some of the data set elements have to be removed with residual analysis, if regression is to deliver satisfactory results. To illustrate the problem let us consider the following example:

Example 1. Imagine an ichthyology research, in which a population of fish is studied. The fish are indiscernible for the attributes such as shape, size, color etc. so one can assume that they belong to one species. The scientists investigate the effect of water temperature on average swimming speed of the fish. After several measurements they obtain a set of data, as depicted in Figure 1, and would like to build a function that links water temperature with fish speed. 


\section{Fish population analysis}



Figure 1

A very rough approach would consist in finding the linear regression function for that data. The function allows us to claim that the speed of fish slowly goes down with the temperature, though the error of the regression is rather high.

There exists however another solution to this problem, namely clustering: after having made a closer look at the measured data one could think of splitting the fish population into two groups. Instead of finding a rough regression function for the whole population, we should rather think in this case of applying clustering, which would result in minimizing the regression indetermination coefficient for each cluster separately. Then we should possibly find a new attribute, which would reflect splitting the fish population into two clusters, thus allowing us to classify the population better.

Therefore, if we assume that examined fish belong to two species, that react differently to temperature changes, we can build not one, but two linear regression functions. For each of these functions the indetermination coefficient can be close to zero, which means that these functions constitute a good model for experimental data. In our example the functions that minimize regression error have been depicted in Figure 2. 




Figure 2

The solution described in the above example does not create problems, if the data set is small and can be manually processed before finding the regression function. We simply split data into two clusters and then compute the regression function for each cluster separately. Such functions would obviously provide us with much better "relationships" between the water temperature and fish speed attributes. Unfortunately in modern data mining applications it happens very seldom that data are small enough, so that one could make the analysis manually. In most cases the whole analysis process must be automated, which makes any human intervention and manual data evaluation impossible.

The goal of the paper is to propose an algorithm, which automatically clusters data set into subsets, so that the regression indetermination coefficients for each subset are minimized. It is not an obvious task, as the regression quality cannot be converted into any kind of distance measure between the elements of the data set, though such a measure is necessary for classical clustering algorithms [1]. Also note that grouping data elements using distance measure (for example Euclidean or Chebyshev) on two-dimensional plane would not help in this case.

We show here how genetic algorithms can be used to solve this problem. We present a simple yet promising genetic clustering algorithm that uses the regression error as a cluster quality measure. The algorithm has been designed for the linear regression based clustering. It has been implemented and tested on data samples, which proved its potential usefulness. Possible extensions and improvements of the algorithm are discussed in the subsequent chapters. 
Section 2 of the paper contains basic definitions of statistical concepts. In Section 3 the proposed algorithm together with sample results are overviewed. In Section 4 we discuss possible algorithm extensions for multiple regression., In Section 5 we discuss an application of the algorithm for planning cellular telephone networks. Finally, Section 6 contains conclusion and further research ideas.

\section{Basic definitions}

We recall here briefly the basic notions referring to the analysis method based on linear regression. For more detailed description see [2] and [3].

Linear regression is a method commonly used for finding a linear dependency between some attributes. It uses statistical data in a form of set of independent variable and dependent variable pairs $\left(x_{i}, y_{i}\right)$, to build a function:

$$
\{(x)=a \cdot x+b
$$

using least squares method.

Finding a linear regression function it is equivalent to finding a solution of two equations:

$$
\begin{aligned}
& \sum_{i=1}^{n} y_{i}=a \sum_{i=1}^{n} x_{i}+n \cdot b \\
& \sum_{i=1}^{n}\left(x_{i} \cdot y_{i}\right)=a \sum_{i=1}^{n} x_{i}^{2}+b \cdot \sum_{i=1}^{n} x_{i}
\end{aligned}
$$

The solution gives us values for the coefficients $a$ and $b$ :

$$
\begin{aligned}
& a=\frac{n \sum_{i=1}^{n} x_{i} \cdot y_{i}-\sum_{i=1}^{n} x_{i} \sum_{i=1}^{n} y_{i}}{n \sum_{i=1}^{n} x_{i}^{2}-\left(\sum_{i=1}^{n} x_{i}\right)^{2}} \\
& b=\frac{\sum_{i=1}^{n} y_{i}}{n}-\frac{\sum_{i=1}^{n} x_{i}}{n} \cdot a
\end{aligned}
$$

In Example 1 the regression function computed this way has been plotted in Figure 1.

The regression quality is measured by the linear indetermination coefficient: 


$$
\varphi^{2}=\frac{\sum_{i=1}^{n}\left(y_{i}-y_{i}\right)^{2}}{\sum_{i=1}^{n}\left(y_{i}-\bar{y}\right)^{2}}, \varphi^{2} \in<0,1>
$$

Referring to Example 1, the indetermination coefficient for the function as on Figure 1is equal to 0.9987 . It means that the two attributes - temperature and speed - have no linear relation.

\section{Algorithm proposal and implementation}

Humans easily do the analysis performed in Example 1, but for data mining purposes we need an automated system that would perform it. We can not use the regression function parameters as a measure of distance between data set items to perform for example k-means clustering or to build a clustering tree (see [1] for classical clustering methods description). We can however use it to compute cluster quality function, in other words a fit function for clusters. Hence to solve our problem we can apply genetic programming approach (see e.g. [4] and [5]), using cluster quality measure as a fitness function.

Below we present a framework of a clustering algorithm, which splits a data set into a predefined number of clusters. If we do not know a priori the number of clusters, we can use the algorithm repeatedly, as in iterative k-means clustering, to find the most effective solution.

To simplify our considerations let us assume that the input set of data has two attributes, and the number of clusters is two. Let $D=\left\{d_{1}, d_{2}, \ldots, d_{n}\right\}$ be the data set containing objects $d=(x, y)$ to be clustered. Let $U$ be the binary vector $\left[u_{1}, u_{2}, \ldots, u_{n}\right]$ representing the distribution of the objects from $D$ into two sets $C_{1}$ and $C_{2}$, so that

$$
\begin{aligned}
& \text { if } u_{i}=0, d_{i} \text { belongs to } C_{l} \\
& \text { if } u_{i}=1, d_{i} \text { belongs to } C_{2}
\end{aligned}
$$

We denote by $P$ the set of all possible vectors and call it objects population.

Now let $R$ be the quadruple $(a, b, c, d)$ representing the coefficients of two linear regression functions $f r_{1}$ and $f r_{2}$ :

$$
\begin{aligned}
& f r_{1}=a x+b \\
& \text { and } \\
& f r_{2}=c x+d
\end{aligned}
$$

Note that the quadruple $R$ can be also represented as a binary vector, by changing coefficient representation into binary numbers. We denote by $Q$ the set of vectors $R$, and call it as function population. 
Using the above definitions we can now write the algorithm for 2 clusters and two-dimensional space of data):

cluster(D):

populate $P$ and $Q$ with random data

repeat

for every vector from $P$ as $U_{i}$

create two data sets $C_{1}$ and $C_{2}$ containing objects from $D$ grouped by $U_{i}$ elements

compute regression functions: $f_{1}$ for objects from $C_{1}$ and $f_{2}$ for objects from $C_{2}$

compute indetermination coefficients $f i_{1}$ and $f i_{2}$ for $f_{1}$ and $f_{2}$ respectively

let $\max \left(f i_{1}, f i_{2}\right)$ be the fit function for $U_{i}$

end;

using the fit functions perform genetic operations on $P$

for the best individual from $P$ compute regression functions $f_{1}$ and $f_{2}$

create individual $R$ using coefficients from $f_{1}$ and $f_{2}$

insert $R$ into $Q$

for every vector from $Q$ as $R_{i}$

for every element from $D$ as $d i$

Let $d i s t_{l}$ be the Euclidean distance from $d_{i}$ to $f r_{l}$ line

Let $d i s t_{2}$ be the Euclidean distance from $d_{i}$ to $f r_{2}$

if dist $_{1}>$ dist $_{2}$ insert $d_{i}$ into $C_{2}$ else insert $d_{i}$ into $C_{l}$

end;

compute the regression functions $f_{1}$ for the objects from $C_{1}$ and $f_{2}$ for the objects from $C_{2}$

estimate indetermination coefficients $f i_{1}$ and $f i_{2}$ for $f_{1}$ and $f_{2}$

let $\max \left(f i_{1}, f i_{2}\right)$ be the fit function for $R_{i}$

end;

using the fit functions perform the genetic operations on $Q$

create individual $U_{x}$ using the $C_{I}$ and $C_{2}$ distributions for the best individual from $Q$

insert $U_{x}$ into $P$

until indetermination coefficients are satisfactory

This algorithm contains effectively two competing populations. One of them, represented by the set $P$ contains individuals that evolve distribution of data set objects between clusters, 
while the second one - represented by the set $Q$ - contains individuals evolving the regression functions. The best individuals are transferred (after conversion) between these two populations. The experiments show that the $Q$ population allows for quick but rough estimation of clusters, while $P$ population fine-tunes the solution.

This algorithm has been implemented in an experimental system and used for clustering small sets of data (200 data objects maximum). Some examples of the results are presented in Figure 3 and Figure 4.



Figure 3 Maximum indetermination coeff. $=0.08$



Figure 4 Maximum indetermination coeff. $=0.35$

\section{Scaling-up the problem into multiple dimensions}

For the practical use of this algorithm, one should be able to cope with objects characterized by more than two attributes. In such a multidimensional space it is much harder to discern linear trends. The multiple regression function has the form of a hyper-plane, thus is more difficult to imagine and visualize. That is why a multidimensional version is expected to reveal lots of interesting knowledge from numerical databases.

In the multiple regression a dependent variable is a vector: 


$$
y=\left[\begin{array}{l}
y_{1} \\
y_{2} \\
\cdots \\
y_{n}
\end{array}\right]
$$

Independent variable $\mathrm{X}$ is a matrix:

$$
X=\left[\begin{array}{cccc}
x_{11} & x_{12} & \ldots & x_{1, k+1} \\
x_{21} & x_{22} & \ldots & x_{2, k+1} \\
\ldots & \ldots & \ldots & \ldots \\
x_{n 1} & x_{n 1} & \ldots & x_{n, k+1}
\end{array}\right]
$$

$\beta$ is a vector of $k$ multiple regression coefficients, $\varepsilon$ is a vector of $n$ random addends. In this case the linear regression function has the form:

$$
\mathrm{y}=\mathrm{X} \beta+\varepsilon
$$

and the estimator of vector $\beta$ is

$$
b=\left(X^{T} X\right)^{-1} X^{T} y
$$

The linear indetermination coefficient has the form:

$$
\phi=\sqrt{\frac{y^{T} y-b^{T} X^{T} y}{y^{T} y-\frac{1}{n}\left(\mathbf{1}^{T} y\right)^{2}}}
$$

Scaling the algorithm may be a difficult task, because with the numbers $k$ and $n$ growing, the calculations on the above matrices, especially matrix inversion, are polynomialy hard. The only way seems to be the use of the regression approximation algorithm [2].

There are plenty of possible applications of the clustering algorithms with multiple regression, not to mention such examples like banking analyses, demographics or engineering. In the next chapter we describe an application, which actually originated the presented idea of clustering. The application is concerned with modeling the traffic in cellular telephone network. 


\section{Applications for cellular network planning}

The idea of using regression in clustering has been inspired by the data mining project performed by a team at Warsaw University of Technology for one of the Polish cellular telecom operators [6]. One of the problems encountered during the implementation of the project was prediction of the traffic for GSM cells.

The network expansion is one of the most important activities for a young cellular telecom provider. It creates a big demand for optimal prediction of the traffic. The operator would like to estimate the number of calls that would be generated over a certain time span on the area where the expansion is planned and new base station are proposed. Such information is crucial for the optimal design of the station equipment - there must be enough transceivers to ensure that every subscriber in the GSM cell created by this station is able to place or receive a call. On the other hand, there should not be too much unused radio channels, because this would mean unnecessary costs.

As a starting point, for our analysis we have extracted geographical information ${ }^{1}$ about the existing GSM cells, together with measured traffic values averaged over certain period. In the company's GIS database a classification schema was used for the terrain types. It was one of the characteristics of the existing cells. The classification was a result of a priori intuition, rather than any statistical or data mining experiments. Roughly the classification contained the following classes.

\{Fields, Forests, Water, (Rocks, Seashores, Swamps), (Roads, Concrete, Parks), Suburbs, Urban, Dense urban, Industrial\}

Using data from GIS, as the first step we have applied multiple regression analysis to estimate traffic value for each cell. As expected, the quality of prediction was quite poor, for the same reasons that were illustrated in Example 1. Therefore we have decided to cluster cells using an extended variant of the algorithm sketched in this paper. The resulting clusters not only allowed us to improve prediction accuracy (now the result reaches $85 \%$ of accuracy with less than 10 clusters), but also helped to find out better classification schema for the cells for subsequent experiments. More details about the experiment may be found in [7].

\section{Conclusion}

The method described in this paper should not be treated as an universal clustering methodology. Partitioning of the data set, achieved by using the algorithm turns out to be useful in special situations, mostly when classical regression analysis gives unsatisfactory results, in spite of the belief that there should be strong correlation between data set elements.

\footnotetext{
${ }^{1}$ In further experiments this data has been enriched with population and economic information, such as average subscriber income etc.
} 
The presented clustering method is probably most useful for mining in numerical data from purely technical applications. It is indeed still a largely unexplored area of automatic knowledge discovery, where our insight about collected data, as well as visualization and manual analysis capabilities are limited. This kind of data mining applications are very different from that experienced in classical, i.e. related to marketing or customer care, data mining [8].

The algorithm presented here is in its first stage of development. First of all it should be extended to include other than linear regression function fitting. Finding a method for automatic selection of the number of clusters would also be very desirable. Improvements have to be done to genetic population representation and fit function, as the currently used Max function is probably not the optimal solution. Finally the genetic operations used in the experimental implementation can be improved.

In [7] another clustering method in the context of linear regression has been proposed. It was an extension to K-means clustering. One of the next steps in our research will also be an evaluation of the two methods and finding out the application areas for them.

\section{Acknowledgements:}

We would like to thank all members of the Data Mining team at Warsaw University of Technology, especially Professor Mieczysław Muraszkiewicz, for help given during the preparation of this paper.

\section{Bibliography}

[1] Duran B.S, Odell P.L : "Cluster Analysis - A Survey", Lecture Notes in Economics and Mathematical Systems, Vol. 100, 1974

[2] Statsoft Inc.: "Electronic Statistics Textbook" Tulsa, 2000, http://www.statsoft.com/textbook/stathome.html

[3] J.Netter, W. Wasserman, G.A. Whitmore, "Applied Statistics", Allyn and Bacon, 1993, pages 531-649

[4] Back T.: "Evolutionary Algorithms in Theory and Practice", Oxford University Press, 1996

[5] Z. Michalewicz: "Genetic Alghorithms + Data Structures = Evolution Programs", Springer Verlag, 1996

[6] W. Daszczuk, P. Gawrysiak, T. Gerszberg, M. Kryszkiewicz, J. Mieścicki, M.

Muraszkiewicz, M. Okoniewski, H. Rybiński, T. Traczyk, Z. Walczak: "Data Mining for

Technical Operation of Telecommunications Companies: a Case Study", submitted to $4^{\text {th }}$

World Multiconference on Systemics, Cybernetics and Informatics (SCI'2000), 2000

[7] P. Gawrysiak, M.Okoniewski: "Applying data mining methods for cellular radio network planning", submitted to Intelligent Information Systems conference (IIS'2000), 2000

[8] Michael J. A. Berry, Gordon Linoff: "Data Mining Techniques: For Marketing, Sales, and Customer Support", John Wiley \& Sons 1997 